Article

\title{
Identification of Leaf-Scale Wheat Powdery Mildew (Blumeria graminis f. sp. Tritici) Combining Hyperspectral Imaging and an SVM Classifier
}

\author{
Jinling Zhao ${ }^{1, *}\left(\mathbb{D}\right.$, Yan Fang ${ }^{2}$, Guomin $\mathrm{Chu}^{2}{ }^{2}$, Hao Yan ${ }^{2}$, Lei $\mathrm{Hu}^{2}$ and Linsheng Huang ${ }^{1, *}$ \\ 1 National Engineering Research Center for Agro-Ecological Big Data Analysis \& Application, \\ Anhui University, Hefei 230601, China \\ 2 School of Electronics and Information Engineering, Anhui University, Hefei 230601, China; \\ P18301127@stu.ahu.edu.cn (Y.F.); P19301110@stu.ahu.edu.cn (G.C.); P19201087@stu.ahu.edu.cn (H.Y.); \\ P19301115@stu.ahu.edu.cn (L.H.) \\ * Correspondence: zhaojl@ahu.edu.cn (J.Z.); linsheng0808@ahu.edu.cn (L.H.)
}

Received: 24 June 2020; Accepted: 22 July 2020; Published: 24 July 2020

\begin{abstract}
Powdery mildew (PM, Blumeria graminis f. sp. tritici) is a devastating disease for wheat growth and production. It is highly meaningful that the disease severities can be objectively and accurately identified by image visualization technology. In this study, an integral method was proposed based on a hyperspectral imaging dataset and machine learning algorithms. The disease severities of wheat leaves infected with PM were quantitatively identified based on hyperspectral images and image segmentation techniques. A technical procedure was proposed to perform the identification and evaluation of leaf-scale wheat PM, specifically including three primary steps of the acquisition and preprocessing of hyperspectral images, the selection of characteristic bands, and model construction. Firstly, three-dimensional reduction algorithms, namely principal component analysis (PCA), random forest (RF), and the successive projections algorithm (SPA), were comparatively used to select the bands that were most sensitive to PM. Then, three diagnosis models were constructed by a support vector machine (SVM), RF, and a probabilistic neural network (PNN). Finally, the best model was selected by comparing the overall accuracies. The results show that the SVM model constructed by PCA dimensionality reduction had the best result, and the classification accuracy reached $93.33 \%$ by a cross-validation method. There was an obvious improvement of the identification accuracy with the model, which achieved an $88.00 \%$ accuracy derived from the original hyperspectral images. This study can provide a reference for accurately estimating the disease severity of leaf-scale wheat PM and other plant diseases by non-contact measurement technology.
\end{abstract}

Keywords: disease severity; hyperspectral imaging; probabilistic neural network; successive projections algorithm; support vector machine; wheat powdery mildew

\section{Introduction}

As one of the main cereal crops, wheat has been widely grown in northern China. In recent years, various wheat diseases, such as powdery mildew (PM, Blumeria graminis f. sp. tritici), stripe rust (Puccinia striiformis f. sp. tritici), and wheat scab (Fusarium graminearum Schwabe), have occurred due to various pathogens and the weather in this region favors the occurrence and spreading of such diseases [1]. The wheat grain yield and quality have been greatly affected, threatening food security. It is becoming increasingly important to assess and control the disease epidemic. When wheat PM occurs, it is important to derive the disease severity from the symptoms, which can provide an essential reference for population virulence and cultivar resistance. Traditionally, more time and labor have been required for phytopathologists to estimate the infection. It is inevitable that a comparison of multiple 
experiments is difficult and some mistakes may be made by humans. With the development of remote sensing and image processing techniques, the quantitative accuracy has been greatly improved $[2,3]$. Ma et al. [4] jointly used the AdaBoost model and a max-relevance and min-redundancy (mRMR) algorithm to detect wheat PM with an overall accuracy of $88.4 \%$. Steddom et al. [5] used a multi-band spectrometer to accurately assess the incidence of brown spot disease in beet, improving the accuracy of brown spot surveillance. Adams et al. [6] monitored soybean chlorosis by studying the spectra of soybean leaves.

After wheat leaves are infected by PM, some symptoms can be visually observed when the severity is relatively serious. Nevertheless, it is usually too late to prevent and control the disease. In fact, some of the infection symptoms can be detected in the near-infrared (NIR) wavelength region rather than the visible range [7]. The PM pathogen can cause changes in the leaf pigment content, cell structure, and leaf water, and the spectral responses differ in different spectral domains accordingly. When the wheat leaves are infected by PM, in comparison with the visible range, the spectral differences are sharper in the NIR wavelength region. Several ground-based remote sensing systems and spectral indices have been developed for plant disease diagnosis and detection [8-12]. For example, Zhang et al. detected the leaf-scale PM of winter wheat using leaf level hyperspectral measurements derived from an Analytical Spectral Device (ASD, Inc., Boulder, CO, USA) spectroradiometer [13]. Shi et al. adopted spectral indices and kernel discriminant analysis to detect and discriminate the pests and diseases in winter wheat using an ASD FieldSpec spectrometer [14]. Non-imaging hyperspectral data are usually used in studies of crop disease monitoring and diagnosis.

More attention has been paid to hyperspectral imaging technology due to its integration of spectra and images. In comparison with visible-band and wide-band images, hyperspectral imaging technology can provide more objective and accurate results due to its integration of images and spectra [15]. It has been widely used in the detection of crop diseases in recent years. Williams et al. [16] successfully detected the fusarium disease on corn by using hyperspectral imaging technology. Bauriege et al. [17] used hyperspectral imaging technology to detect early wheat Fusarium diseases, and the accuracy of disease level detection was $87 \%$. Del Fiore et al. [18] conducted early detection research on corn fungal diseases using hyperspectral imaging technology. Liu et al. [19] established a model for investigating the relationship between the severity of rice blight and the spectrum based on a spectral study of rice ears. Graeff et al. [20] analyzed the spectra of wheat leaves and found that powdery mildew had a strong spectral response in some sensitive bands. Therefore, hyperspectral imaging can provide a fine comparison of healthy and diseased wheat plants.

After being infected by PM, pustules with a light white (sometimes light yellow) color can be observed on wheat leaves [21]. The significant spectral differences between healthy leaves and infected ones can be accurately identified by selecting pure regions of interest (ROIs) on high-resolution hyperspectral images. Comprehensive information combining the visible and NIR wavelengths can provide a more rational and accurate diagnosis of the disease. In addition, a leaf-scale diagnosis study is an appropriate way of investigating the disease features compared with canopy and regional scales. Therefore, the objectives of this study were (1) to examine the effect on identifying characteristic bands before and after spectral smoothing during the acquisition of hyperspectral data, (2) to compare the three-dimensional reduction methods for selecting spectral bands sensitive to wheat PM, and (3) to identify the severity levels of PM on wheat leaves by comparing the classification accuracies of three inversion models.

\section{Materials and Methods}

\subsection{Technical Procedure}

Three primary steps were required to carry out the technical procedure (Figure 1). The original hyperspectral images of healthy and diseased wheat leaves were first smoothed by the Savitzky-Golay (S-G) filter, and the spectral and image features were then identified. To reduce the calculation time 
and highly-significant correlation among neighboring bands, three-dimensional reduction methods were comparatively used, namely principal component analysis (PCA), random forest (RF), and the successive projections algorithm (SPA). The average spectra of original and dimensionally-reduced wheat leaves were extracted by multiple scattering corrections (MSC). After removing the regions of thumb tacks, the disease spots on the leaves were extracted in the 2R-G-B color difference RGB color space. The disease severity for each leaf was calculated by the ratio between disease spots and the entire leaf. Considering the spectral and corresponding disease severity simultaneously, three detection models were comparatively constructed by the support vector machine (SVM), RF, and the probabilistic neural network (PNN).

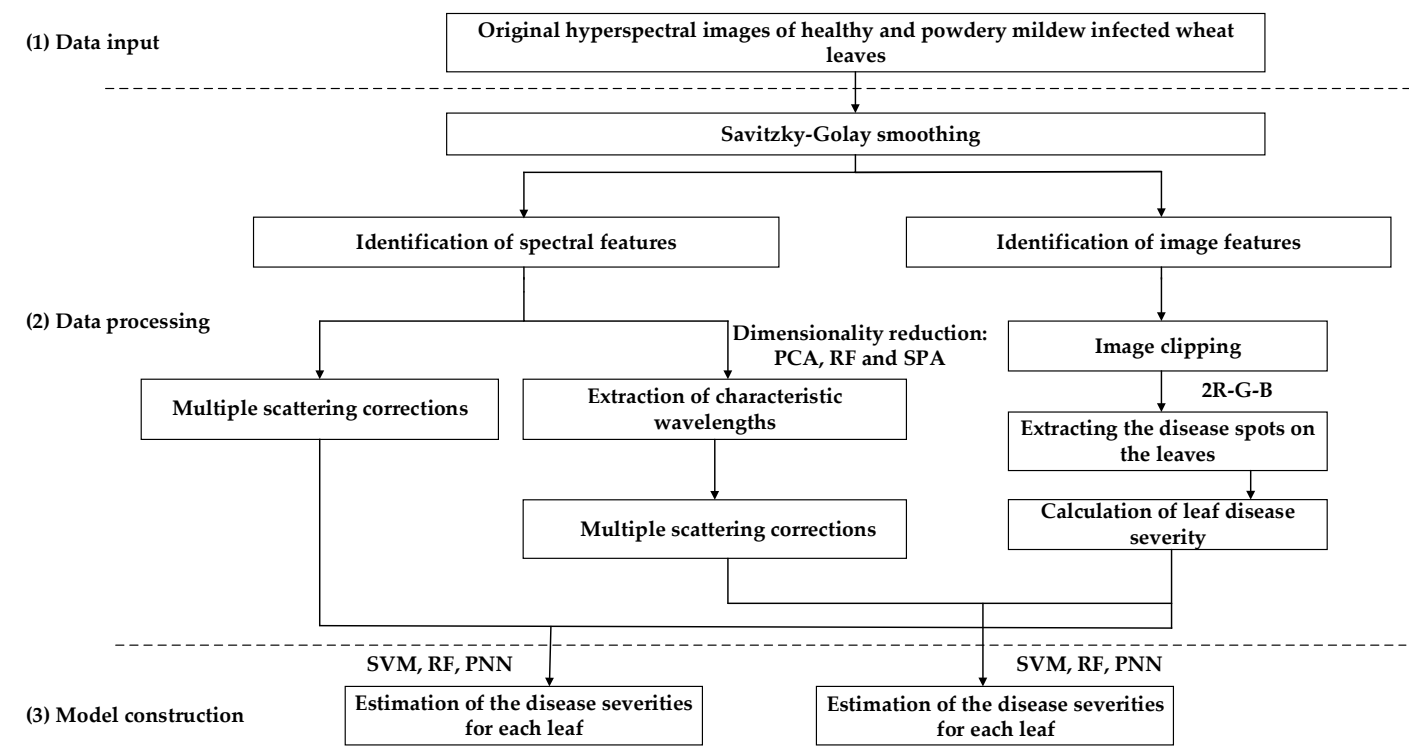

Figure 1. The overall technical workflow of the study.

\subsection{Acquision and Preprocessing of Hyperspectral Images}

\subsubsection{Experimental Design}

The experiment was carried out at the experimental field of Beijing Academy of Agriculture and Forestry Sciences $\left(39.93^{\circ} \mathrm{N}, 116.27^{\circ} \mathrm{E}\right)$, China [22]. To ensure disease incidence, the wheat variety Jingdong-12 susceptible to PM was selected and grown under normal water and fertilizer management. The symptoms could be gradually observed with the increase of disease severities and the development of growth stages. To improve the contrast enhancement between healthy and diseased wheat leaves, data collection was performed during the grain-filling stage, which is an essential period that affects the yield and quality of wheat. Wheat plants were first assessed by an experienced pathologist in the field, without destroying them. A total of 75 sample leaves, including 60 diseased leaves and 15 healthy leaves, were picked. To keep the wheat leaves fresh, a portable fridge was used to store them. In addition, the hyperspectral imaging device was installed in a dark room near the experimental site and fixed over a sampling platform covered with a black cloth. Leaves with different severities were fixed on the cloth by thumb tacks.

\subsubsection{Acquisition of Hyperspectral Image Cubes}

Three necessary procedures had to be performed to acquire and process the hyperspectral images, including mosaicing, reflectance conversion, and spectral smoothing (Figure 2) [23]. A ground-based pushbroom imaging spectrometer (PIS) was used to collect the hyperspectral images. PIS acquires images by linear array pushbroom imaging technology. It was jointly developed by the Beijing Research Center for Information Technology in Agriculture and the University of Science and Technology of 
China. The sensor can collect a hyperspectral image (cube) and pixel-by-pixel spectral information within the effective wavelengths of 400-1000 nm, with a spectral resolution of $2 \mathrm{~nm}$ and a sampling interval of $0.7 \mathrm{~nm}$. It has a field of view of $16^{\circ}$ and a spatial resolution of $5-10 \mathrm{~mm}$. A hyperspectral image of 1400 (spatial dimension) $\times 1024$ (spectral dimension) can be acquired for each scan. There are various illumination intensities in different wavelengths, due to the existence of dark current. Some noises can be caused in the low-illumination spectral bands. To reduce the noises and perform reflectance conversion, the standard reference panel must be used to optimize the instrument before and after collecting the spectra.

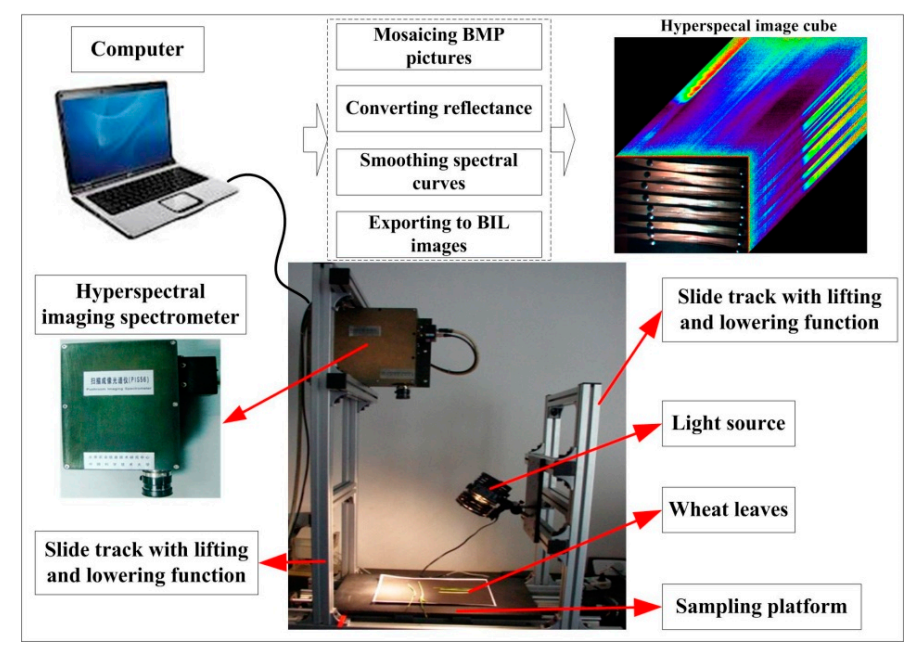

Figure 2. The devices used to measure the hyperspectral reflectance of wheat leaves.

\subsubsection{Data Preprocessing}

When the Bitmap (BMP) format pictures were mosaiced to generate an entire image of a group of wheat leaves, reflectance conversion was conducted using Equation (1). Last but not least, to reduce the random noises during data collection, the S-G filter was used to improve the spectral smoothness in the Environment for Visualizing Images (ENVI). Firstly, the spectral mean values of 75 hyperspectral leaves were extracted by calculating all of the pixel values. The derivative function was used to correct the baseline effects, which could amplify and resolve the overlapped signal. In SG smoothing, the window size and polynomial order must be specified. The window size must be an odd number and was set to 21 here, and the polynomial order must be less than the window length and was set to 2 in our experiment [24]. A comparison of the spectral curves before and after the S-G smoothing filter is shown in Figure 3.

$$
\rho=\mathrm{a} \times D N+\mathrm{b},
$$

where $\rho$ is the spectral reflectance, $a$ and $b$ are the coefficients, and DN is the digital number for $a$ pixel in the original image. The values of $a$ and $b$ can be ensured by the least-squares method when incorporating the measured spectral value and corresponding DN into Equation (1). The $\rho$ of the hyperspectral image can be calculated accordingly. 

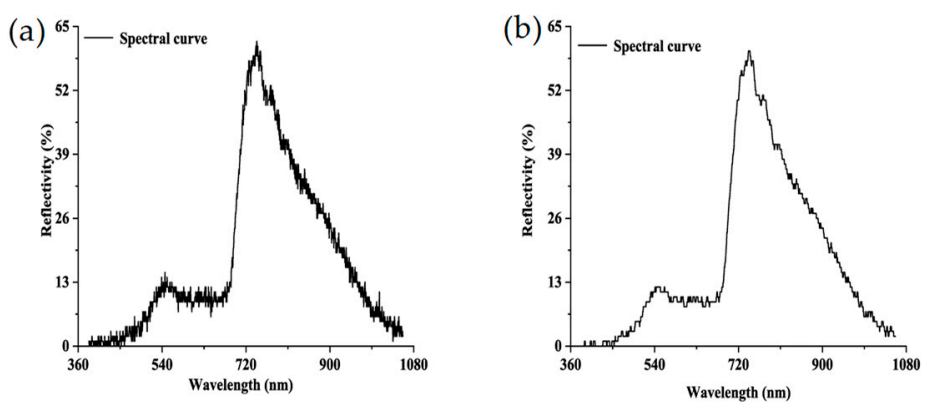

Figure 3. Comparison of hyperspectral reflectance curves between (a) original and (b) Savitzky-Golay (S-G) filter-smoothed data.

\subsubsection{Determination of Disease Severity}

According to the rules for the investigation and forecasting of wheat PM of China (NY/T 613-2002), the disease severity (DS) can be divided into eight levels based on the ratio of diseased spots to total leaf area (Equation (2)), i.e., 1\%, 5\%, 10\%, 20\%, 40\%, 60\%, 80\%, and $100 \%$. Nevertheless, the spectral differences are not significant enough for some levels, especially for neighboring levels. In our study, to enhance the spectral comparison of different levels, the eight levels were recategorized into three levels: Healthy (Level 0, DS $<5 \%$ ), slight (Level 1, 5\% $<$ DS $<40 \%$,), and serious (Level 2, DS $>40 \%$ ). The disease spots were identified using the threshold segmentation method and are shown in the 2R-G-B color space (Figure 4). It is obvious that the model can identify the disease spots well.

$$
D=\frac{A_{\mathrm{D}}}{A_{\mathrm{T}}} \times 100 \%
$$

Here, $D$ is the disease severity of the wheat leaf, $A_{\mathrm{D}}$ is the area of PM disease spots on the leaf, and $A_{\mathrm{T}}$ is the total area of the leaf.

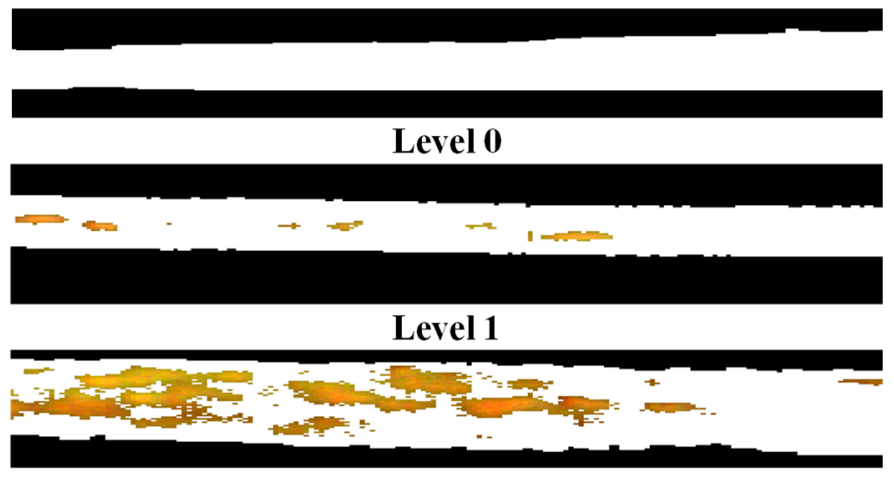

\section{Level 2}

Figure 4. The segmentation results of disease spots for the three levels.

\subsection{Methods for Hyperspectral Dimensionality Reduction}

The original hyperspectral image ranges from 400 to $1000 \mathrm{~nm}$ and is divided into 1024 bands. When all of the bands are used as the input variables, a long computation time and advanced computer configuration are required. In addition, in such a high-dimensional space, it is inevitable that there is a strong correlation among multiple bands. The information redundancy and random noises will affect the model sensitivity and reduce the identification accuracy $[25,26]$. In general, there is no universal dimensionality reduction method for any application scene. Consequently, it is highly necessary to select the wavelength bands that are sensitive to PM. In this study, three methods were comparatively used, namely principal component analysis (PCA), random forest (RF), and the successive projections algorithm (SPA). 


\subsubsection{PCA}

PCA is a method that removes redundant information between bands and compresses multi-band image information to a few bands that are more effective than the original bands. It is a statistical method widely used in unsupervised dimensionality reduction [27]. Linear transformation is used to transform the original data into a set of linearly independent representations for each dimension, which can be used to extract the main feature components of the objective data. In essence, the direction with the largest variance is used as the main feature to make sure that there are no correlations in different orthogonal directions $[28,29]$. Fewer new variables are selected to replace the original variables under the premise of keeping most of the spectral information. In this study, the first three principal components contained $99.21 \%$ of the variance information, so they were retained. The peaks and valleys were selected as the characteristic wavelength bands.

\subsubsection{SPA}

The successive projections algorithm (SPA) is an effective variable-selection technique that has attracted increasing interest in hyperspectral remote sensing [30-32]. The primary purpose is to select wavelengths in which the information content is minimally redundant to solve collinearity problems. It is a forward selection method starting with one wavelength, and then incorporates a new one at each iteration, until a specified number $N$ of wavelengths is reached [33]. The specific steps of the SPA are shown in Table 1.

Table 1. Primary steps for performing the successive projections algorithm (SPA)-based dimensionality reduction method.

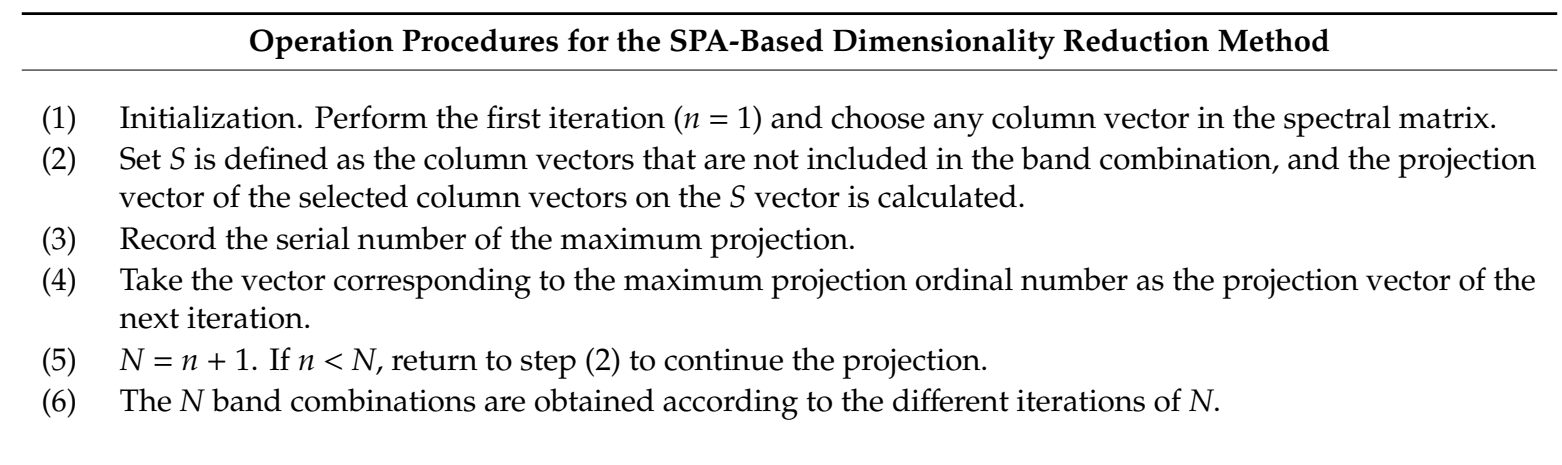

\subsection{Modeling Methods}

The seventy-five samples were divided into five groups. A group was selected as the test set and the remaining four groups were used as the training set. Three detection models using the three-dimensional reduction methods were constructed and compared. Cross-validation was used to evaluate the accuracy of the constructed models. The ROI tool in ENVI was used to create ROIs to verify the accuracy of disease spot segmentation.

\subsubsection{SVM}

The SVM is a machine learning method based on the statistical learning theory [34,35]. It can maximize the separation or margin between samples of different classes by constructing a set of hyperplanes. There are several unique advantages in addressing issues such as small samples, non-linearity, and high-dimensional pattern recognition problems. The phenomena of "dimensional disaster" and "over-learning" can be avoided to a great degree. Several parameters should be evaluated and specified, including the kernel function, values for gamma, and cost. In this study, the radial basis 
function (RBF) (Equation (3)) was used as the kernel function. The cross-validation was applied to identify the best parameters.

$$
\mathrm{K}\left(x_{i}, x\right)=\exp \left(-\frac{\left\|x-x_{i}\right\|}{2 \sigma^{2}}\right)
$$

Here, $x_{i}$ is the input training data and $\sigma$ is the kernel parameter.

\subsubsection{RF}

$\mathrm{RF}$, consisting of multiple decision trees, is a classification or regression method. It was proposed by Leo Breiman in 2001 [36]. The decision trees are created on a bootstrap sample of training data by using a random selection of variable subsets. The $n$ training sets can be obtained after n-time sampling. The $n$ decision tree models are constructed based on each new training set and a random forest can be generated. Every tree of the forest then votes to determine the sample's class, and a majority vote makes the final decision $[37,38]$. In our study, the RF classifier was built with the recommended values by Breiman, with the number of decision trees being 500 and default values for other parameters.

\subsubsection{PNN}

A probabilistic neural network (PNN) is a direct continuation of the work on Bayes classifiers [39]. It is a branch of radial basis function networks that belongs to a feedforward neural network. There are four layers, including input units, pattern units, summation units, and output units. The nodes are allocated in the three layers after the inputs. There is one pattern node for each training example. Each summation node receives the outputs from pattern nodes associated with a given class. The output nodes are binary neurons that produce the classification decision. More precisely, the PNN is interpreted as a function that approximates the probability density of the underlying examples' distribution, rather than the examples directly by fitting [40]. A Gaussian function (Equation (4)) is used to connect the input layer and pattern layer. The matching degree is calculated between neurons of the two layers. Then, the specific class of input samples can be ensured by cumulatively adding and averaging the matching degree for each category.

$$
y_{g}(x ; \sigma)=\frac{1}{l_{g}(2 \pi)^{n / 2} \sigma^{n}} \sum_{i=1}^{l_{g}} \exp \left(-\sum_{j=1}^{n} \frac{\left(x_{i j}(g)-x_{j}\right)^{2}}{2 \sigma^{2}}\right)
$$

Here, $l_{g}$ is the number of category $g ; n$ is the feature number; $\sigma$, the unique adjustable factor, is the smoothing parameter located between 0 and 1 ; and $x_{i j}$ is the $j$ th datum of the $i$ th neuro for $g$.

\section{Results and Discussion}

\subsection{Characterizaion of the Disease Severities}

The red $(680 \mathrm{~nm})$, green $(550 \mathrm{~nm})$, and blue $(450 \mathrm{~nm})$ bands were composited to show the pseudo-color image in ENVI (Figure 5). The comparison of three disease severities of image (Figure 5a) and spectral (Figure 5b) features can be observed. When checking the image features, it can be found that the leaf of Level 0 has a homogeneous color and texture. For Level 1, the increase of spores affects the textural structure of the original leaf and scattered speckles can be found. The leaf of Level 2 has the highest textural complexity, with more yellow or light-yellow speckles appearing on the leaf. Considering the spectral features, the overall trends of the average spectral reflectivity of the three severities are similar during the specified wavelength bands. Several reflectance peaks for the three levels can be found in the wavelength bands of 555-760 nm. In comparison with Level 2, the spectral curves for Level 0 and Level 1 are extremely similar. Nevertheless, their spectral responses differ in the visible and NIR spectral regions. With the increase of disease severity, the reflectivity also has a large value from 460 to $710 \mathrm{~nm}$. Conversely, the reflectivity shows the opposite trend within the spectral range of 730 to $900 \mathrm{~nm}$. 


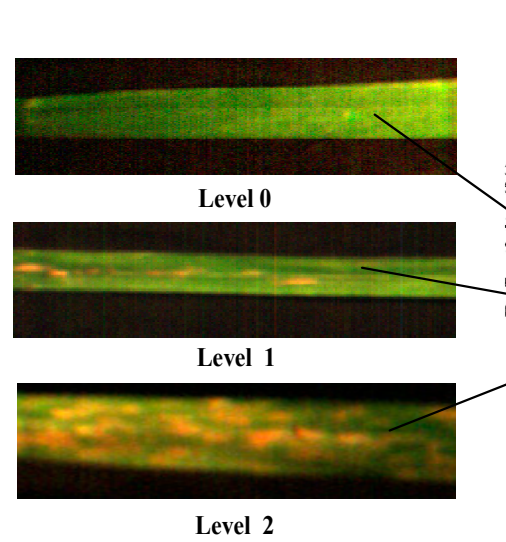

(a)

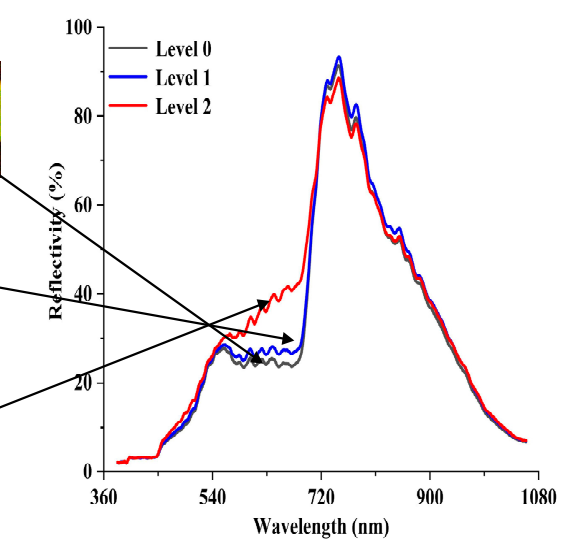

(b)

Figure 5. (a) False-color composite image showing the three levels and (b) Savitzky-Golay (S-G) filter-smoothed data.

A hyperspectral spectrometer can collect information based on the measurement of reflected solar energy in hundreds of narrow wavelengths in the human visible spectrum, and also in the NIR and shortwave infrared (SWIR) regions of the solar spectrum. We assume that non-imaging hyperspectral data generally consist of a mixed spectrum in a certain region because of the influences of the background, shadow, soil, etc. Conversely, imaging spectroscopy can simultaneously capture the spectral and image information of materials on the surface. In comparison with high-altitude satellite imagery, ground-based hyperspectral imaging data usually have high spectral and spatial resolutions, which can be very useful in precisely detecting various stresses in green vegetation [24,41]. Additionally, a leaf-scale hyperspectral image is superior in terms of characterizing the PM disease for the canopy-scale datum, due to its simple background. Corresponding band selection and modeling methods can provide a reference for disease detection at the canopy, field, and regional scale. Nevertheless, some issues must be addressed during the processing and application of the hyperspectral dataset, especially the hyperspectral imaging data, such as through feature selection, dimensionality reduction, and sensitivity analysis [29,30,42].

\subsection{Selection of the Sensitive Spectral Bands}

Comparisons of selected sensitive spectral bands by the three-dimensional reduction methods are shown in Figure 6 and Table 2. A total of 12 characteristic bands was obtained by the PCA method, and these bands are primarily located around the spectral range between 500 and $850 \mathrm{~nm}$ (Figure 6a). A total of 16 characteristic bands were identified through the RF method, among which 11 bands are located between 590 and $700 \mathrm{~nm}$ (Figure 6b). In comparison with the above two methods, only 10 bands were selected by the SPA method, and most of them are distributed between 500 and $760 \mathrm{~nm}$ (Figure 6c). Considering the selected characteristic bands derived from the three-dimensional reduction methods, it can be found that most of the bands sensitive to wheat PM are located in the visible light range. Nevertheless, there is a significant difference for the band selection of the three methods. For the RF and SPA methods, only one and two bands were located in the NIR spectral range. Conversely, a total of five characteristic bands were selected for the PCA method. 


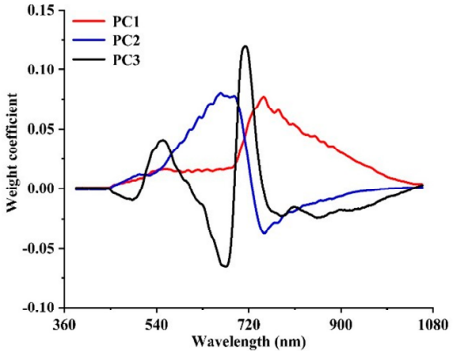

(a) PCA

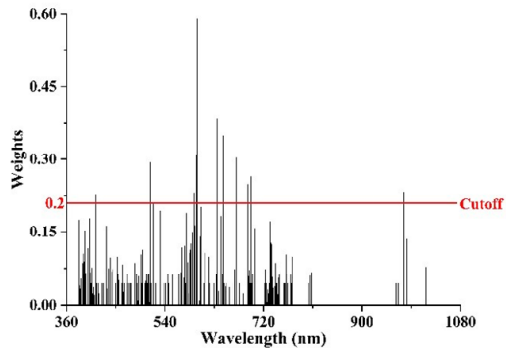

(b) RF

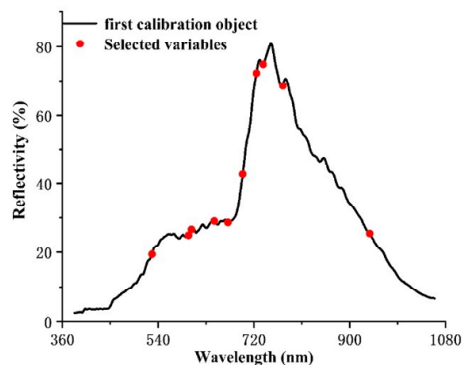

(c) SPA

Figure 6. Comparison of characteristic band selection using (a) principal component analysis (PCA), (b) random forest (RF), and (c) the SPA.

Table 2. Selected sensitive bands derived from PCA, RF, and the SPA.

\begin{tabular}{cc}
\hline Method & Sensitive Band (nm) \\
\hline PCA & $492.7,551.5,665.2,675.8,713.4,749.1,750.5,769.6,778.2,783.5,808.6,853.6$ \\
RF & $413.3,513.2,519.1,592.5,593.1,595.1,597.1,598.4,605,605.7,635.4,646.7,691.6,696.9,737.2,976.5$ \\
SPA & $423.9,528.4,597.1,602.4,645.4,675.1,714.1,737.2,774.3,1057.8$ \\
\hline
\end{tabular}

To compare the band selection of PCA, RF, and the SPA, a correlation analysis is shown in Figure 7. It was found that the sensitive bands can be more easily selected by the PCA method (Figure 7a). The characteristic bands selected by the RF method are highly correlated, specifically with 13 wavelengths between 513.2 and $696.9 \mathrm{~nm}$ (Figure $7 \mathrm{~b}$ ). The figure shows that the correlation between adjacent bands is higher than that of separable bands, which may be approximately continuous and transferable [43]. The correlation between the characteristic bands obtained by the SPA method is minimal (Figure $7 \mathrm{c}$ ). The reason for this is that the principle of the SPA is to find the minimum set of redundant information variables from $X$ variables, where the selected bands are the group with the lowest correlation between each other [33]. These wavelengths range from 423.9 to $1057.8 \mathrm{~nm}$, with the largest span and the lowest accuracy for the three classifiers, showing that the 10 characteristic bands do not respond strongly to wheat PM.

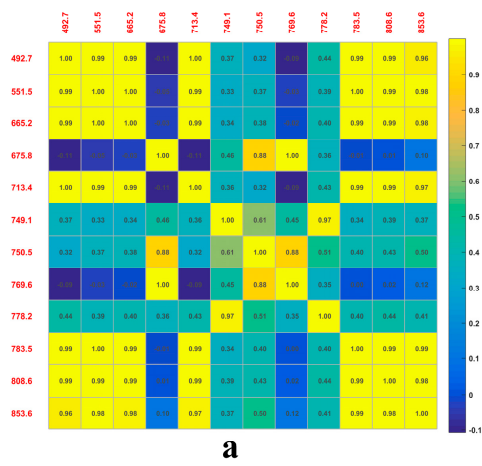

Figure 7. Wavelength correlation for three-dimensional reduction methods:

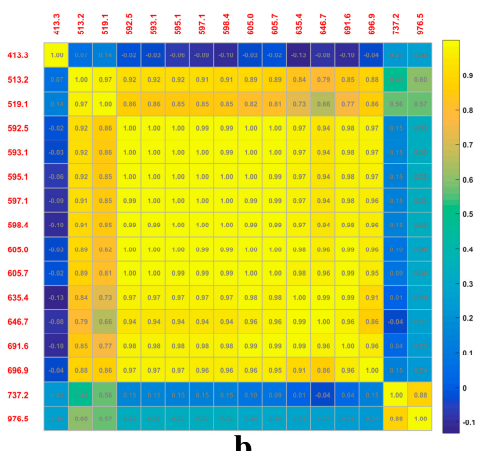

b

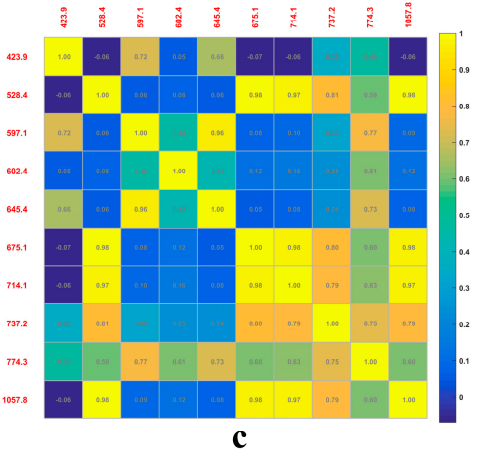

(a) PCA; (b) RF; (c) SPA.

\subsection{Models for Identifying Disease Spots on the Leaves}

The comparison of classification accuracies is shown in Table 3. The results show that there are no significant differences among the SVM, RF, and PNN models constructed using the original hyperspectral imagery. When comparing the accuracies of the three-dimensional reduction-based models, we can find that the PCA-based SVM model has the highest accuracy of 93.33\%. A 5.33\% increase is achieved by the dimensionality reduction of hyperspectral bands. The RF model has the same accuracy of $92.00 \%$ for the three-dimensional reduction methods, showing that RF has a relatively stable performance. Considering the PNN modeling method, there is no significant accuracy increase 
for the three-dimensional reduction methods. All of the accuracies are lower than $90.00 \%$ and the highest one is just $89.33 \%$.

Table 3. Comparison of identification accuracies for the support vector machine (SVM), RF, and the probabilistic neural network (PNN).

\begin{tabular}{cccc}
\hline Method & SVM & RF & PNN \\
\hline None & $88.00 \%$ & $88.00 \%$ & $86.67 \%$ \\
PCA & $93.33 \%$ & $92.00 \%$ & $89.33 \%$ \\
RF & $88.00 \%$ & $92.00 \%$ & $89.33 \%$ \\
SPA & $88.00 \%$ & $92.00 \%$ & $88.00 \%$ \\
\hline
\end{tabular}

The running time of the three models was also compared, in addition to the classification accuracies. It was found that the three methods have a significant difference in running time for the same sample data. As can be seen in Table 4, the RF model is the fastest and takes only 0.39 s, while the SVM model requires $9.91 \mathrm{~s}$. Conversely, the PNN model takes the longest time, with the running time of $2327.80 \mathrm{~s}$.

Table 4. Comparison of the running time for the SVM, RF, and the PNN.

\begin{tabular}{cccc}
\hline Method & SVM & RF & PNN \\
\hline Running time (s) & 9.91 & 0.39 & 2327.80 \\
\hline
\end{tabular}

In general, some factors can affect the identification efficiency of wheat PM using hyperspectral imaging, such as the sample quantity, feature selection algorithms, and dimension reduction methods. For example, they usually require more powerful computers and longer running times because of the large data volume and high-dimensional features required for hyperspectral imaging data [44]. Some dimension reduction methods and effective classifiers need to be adopted. In our study, three dimension reduction methods, including PCA, RF, and the SPA, and three classifiers, including the SVM, RF, and the PNN, were selected. It was found that the identification accuracy (Table 3) and running time (Table 4) can be improved by different dimensionality reduction methods, but there are significant differences in the improvement degree.

In terms of the differences of the running time of the three models, the reason for this may be related to the PNN's four-layer transmission structure [45]. The training and testing of the RF classifier are relatively efficient because the algorithm is a multi-classifier, which can avoid training the classifier multiple times and comprises a small amount of calculation. The applicability and sensitivity must be first evaluated by different dimension reduction methods [46-48]. In addition to data processing algorithms and classifiers, the separability of PM leaves with different severities is an important influencing factor. Transitional samples between two levels are easily misclassified. In this study, $45 \%$ of the infected area of a leaf was taken as a critical value, and the test could be easily misclassified near the critical value. For example, Levels 0 and 1 are easily misclassified, because it is usually hard to discriminate the differences between a healthy leaf and a slightly infected leaf. The sample quantity is also an affecting factor. When the number of samples per level is small and the number of training samples is small, it is difficult to improve the identification accuracy.

\section{Conclusions}

The PM infection of wheat plants changes the leaf pigment concentration, cell structure, water content, etc., which provides a physical mechanism allowing the detection of such a disease using hyperspectral imaging. Three-dimensional reduction methods, including PCA, RF, and the SPA, and three classifiers, including the SVM, RF, and the PNN, were used to comparatively identify the leaf-scale wheat PM. Consequently, the disease models obtained the highest accuracies for the three classifiers using the PCA dimension reduction method. The SVM model had the highest accuracy, 
while the RF model obtained stable identification results. Conversely, the PNN model also exhibited a relatively stable performance, but all of the accuracies were lower. In addition to the identification accuracy, we also considered the running time for each model. The PCA-based SVM model had the best performance in terms of detecting leaf-scale wheat PM after comprehensively considering the identification accuracy and running time. This investigation can provide a case study for accurately and finely detecting wheat PM and can also provide a band selection for developing a portable hyperspectral spectrometer.

Author Contributions: Conceptualization, J.Z.; methodology, Y.F.; formal analysis, G.C.; investigation, Y.F.; data curation, L.H. (Lei Hu) and H.Y.; writing-original draft preparation, Y.F.; writing-review and editing, J.Z. and L.H. (Linsheng Huang); funding acquisition, J.Z. All authors have read and agreed to the published version of the manuscript.

Funding: This research was funded by [National Natural Science Foundation of China] grant number [31971789]. And the APC was funded by [National Natural Science Foundation of China].

Acknowledgments: This work was supported by the National Natural Science Foundation of China (31971789, 61672032), Natural Science Foundation of Anhui Province (2008085MF184), and Natural Science Research Project of Anhui Provincial Education Department (KJ2019A0030).

Conflicts of Interest: The authors declare no conflicts of interest.

\section{References}

1. Duveiller, E.R.; Singh, P.; Nicol, J.M. The challenges of maintaining wheat productivity: Pests, diseases, and potential epidemics. Euphytica 2007, 157, 417-430. [CrossRef]

2. Nilsson, H. Remote sensing and image analysis in plant pathology. Annu. Rev. Phytopathol. 1995, 33, 489-528. [CrossRef] [PubMed]

3. Zhang, J.; Huang, Y.; Pu, R.; Gonzalez-Moreno, P.; Yuan, L.; Wu, K.; Huang, W. Monitoring plant diseases and pests through remote sensing technology: A review. Comput. Electron. Agric. 2019, 165, 104943. [CrossRef]

4. Ma, H.Q.; Huang, W.J.; Jing, Y.S.; Dong, Y.Y.; Zhang, J.C.; Nie, C.W.; Tang, C.C.; Zhang, J.L.; Huang, L.S. Remote sensing monitoring of wheat powdery mildew based on AdaBoost model combining mRMR algorithm. Trans. Chin. Soc. Agric. Eng. 2017, 33, 162-169.

5. Steddom, K.; Bredehoeft, M.W.; Khan, M.; Rush, C.M. Comparison of visual and multispectral radiometric disease evaluations of Cercospora leaf spot of sugar beet. Plant Dis. 2005, 89, 153-158. [CrossRef] [PubMed]

6. Adams, M.L.; Philpot, W.D.; Norvell, W.A. Yellowness index: An application of spectral second derivatives to estimate chlorosis of leaves in stressed vegetation. Int. J. Remote Sens. 1999, 20, 3663-3675. [CrossRef]

7. Zhang, J.C.; Yuan, L.; Wang, J.H.; Huang, W.J.; Chen, L.P.; Zhang, D.Y. Spectroscopic leaf level detection of powdery mildew for winter wheat using continuous wavelet analysis. J. Integr. Agric. 2012, 11, 1474-1484. [CrossRef]

8. Bravo, C.; Moshou, D.; West, J.; McCartney, A.; Ramon, H. Early disease detection in wheat fields using spectral reflectance. Biosyst. Eng. 2003, 84, 137-145. [CrossRef]

9. Moshou, D.; Bravo, C.; West, J.; Wahlen, S.; McCartney, A.; Ramon, H. Automatic detection of 'yellow rust' in wheat using reflectance measurements and neural networks. Comput. Electron. Agric. 2004, 44, 173-188. [CrossRef]

10. Huang, W.; Guan, Q.; Luo, J.; Zhang, J.; Zhao, J.; Liang, D.; Huang, L.; Zhang, D. New optimized spectral indices for identifying and monitoring winter wheat diseases. IEEE J. STARS 2014, 7, 2516-2524. [CrossRef]

11. Huang, W.J.; David, W.L.; Niu, Z.; Zhang, Y.J.; Liu, L.Y.; Wang, J.H. Identification of yellow rust in wheat using in-situ spectral reflectance measurements and airborne hyperspectral imaging. Precis. Agric. 2007, 8, 187-197. [CrossRef]

12. Ashourloo, D.; Mobasheri, M.R.; Huete, A. Developing two spectral disease indices for detection of wheat leaf rust (Puccinia triticina). Remote Sens. 2014, 6, 4723-4740. [CrossRef]

13. Zhang, J.C.; Pu, R.L.; Wang, J.H.; Huang, W.J.; Yuan, L.; Luo, J.H. Detecting powdery mildew of winter wheat using leaf level hyperspectral measurements. Comput. Electron. Agric. 2012, 85, 13-23. [CrossRef]

14. Shi, Y.; Huang, W.J.; Luo, J.H.; Huang, L.S.; Zhou, X.F. Detection and discrimination of pests and diseases in winter wheat based on spectral indices and kernel discriminant analysis. Comput. Electron. Agric. 2017, 141, 171-180. [CrossRef] 
15. Bohnenkamp, D.; Kuska, M.T.; Mahlein, A.K.; Behmann, J. Hyperspectral signal decomposition and symptom detection of wheat rust disease at the leaf scale using pure fungal spore spectra as reference. Plant Pathol. 2019, 68, 1188-1195. [CrossRef]

16. Williams, P.J.; Geladi, P.; Britz, T.J.; Manley, M. Investigation of fungal development in maize kernels using NIR hyperspectral imaging and multivariate data analysis. J. Cereal Sci. 2012, 55, 272-278. [CrossRef]

17. Bauriegel, E.; Giebel, A.; Geyer, M.; Schmidt, U. Early detection of Fusarium infection in wheat using hyperspectral imaging. Comput. Electron. Agric. 2011, 75, 304-312. [CrossRef]

18. Del Fiore, A.; Reverberi, M.; Ricelli, A.; Pinzari, F.; Serranti, S.; Fabbri, A.A.; Bonifazi, G.; Fanelli, C. Early detection of toxigenic fungi on maize by hyperspectral imaging analysis. Int. J. Food Microbiol. 2010, 144, 64-71. [CrossRef]

19. Liu, Z.Y.; Wu, H.F.; Huang, J.F. Application of neural networks to discriminate fungal infection levels in rice panicles using hyperspectral reflectance and principal components analysis. Comput. Electron. Agric. 2010, 72, 99-106. [CrossRef]

20. Graeff, S.; Link, J.; Claupein, W. Identification of powdery mildew and take-all disease in wheat by means of leaf reflectance measurements. Cent. Eur. J. Biol. 2006, 1, 275-288.

21. Rémus-Borel, W.; Menzies, J.G.; Bélanger, R.R. Silicon induces antifungal compounds in powdery mildew-infected wheat. Physiol. Mol. Plant Pathol. 2005, 66, 108-115. [CrossRef]

22. Huang, L.S.; Ding, W.J.; Liu, W.J.; Zhao, J.L.; Huang, W.J.; Xu, C.; Liang, D. Identification of wheat powdery mildew using in-situ hyperspectral data and linear regression and support vector machines. J. Plant Pathol. 2019, 101, 1035-1045. [CrossRef]

23. Zhao, J.L.; Huang, W.J.; Zhang, D.Y.; Luo, J.H.; Zhang, J.C.; Huang, L.S.; Chen, S. Characterization and identification of leaf-scale wheat powdery mildew using a ground-based hyperspectral imaging system. Disaster Adv. 2012, 5, 1657-1662.

24. Yang, X.L.; Hong, H.M.; You, Z.H.; Cheng, F. Spectral and image integrated analysis of hyperspectral data for waxy corn seed variety classification. Sensors 2015, 15, 15578-15594. [CrossRef]

25. Liu, Y.; Xie, H.; Chen, Y.H.; Tan, K.Z.; Wang, L.G.; Xie, W. Neighborhood mutual information and its application on hyperspectral band selection for classification. Chemometr. Intell. Lab. 2016, 157, 140-151. [CrossRef]

26. Wang, Y.Q.; Lv, Y.J.; Liu, H.; Wei, Y.G.; Zhang, J.W.; An, D.; Wu, J.W. Identification of maize haploid kernels based on hyperspectral imaging technology. Comput. Electron. Agric. 2018, 153, 188-195. [CrossRef]

27. Abdi, H.; Williams, L.J. Principal component analysis. Wires. Computation. Stat. 2010, 2, 433-459. [CrossRef]

28. Genuer, R.; Poggi, J.; Tuleaumalot, C. Variable selection using random forests. Pattern Recogn. Lett. 2010, 31, 2225-2236. [CrossRef]

29. Ye, Y.; Wu, Q.; Huang, J.Z.; Ng, M.K.; Li, X. Stratified sampling for feature subspace selection in random forests for high dimensional data. Pattern Recogn. 2013, 46, 769-787. [CrossRef]

30. Wu, D.; He, Y.; Shi, J.; Feng, S. Exploring near and midinfrared Spectroscopy to Predict Trace Iron and Zinc Contents in Powdered Milk. J. Agric. Food Chem. 2009, 57, 1697-1704. [CrossRef]

31. Assis, C.; Oliveira, L.S.; Sena, M.M. Variable selection applied to the development of a robust method for the quantification of coffee blends using mid infrared spectroscopy. Food Anal. Method. 2018, 11, 578-588. [CrossRef]

32. Liu, F.; Jiang, Y.; He, Y. Variable selection in visible/near infrared spectra for linear and nonlinear calibrations: A case study to determine soluble solids content of beer. Anal. Chim. Acta 2009, 635, 45-52. [CrossRef] [PubMed]

33. Araújo, M.C.U.; Saldanha, T.C.B.; Galvao, R.K.H.; Yoneyama, T.; Chame, H.C.; Visani, V. The successive projections algorithm for variable selection in spectroscopic multicomponent analysis. Chemometr. Intell. Lab. 2001, 57, 65-73. [CrossRef]

34. Cortes, C.; Vapnik, V. Support-vector networks. Mach. Learn. 1995, 20, 273-297. [CrossRef]

35. Boser, B.E.; Guyon, I.M.; Vapnik, V.N. A training algorithm for optimal margin classifiers. In Proceedings of the Fifth Annual Workshop on Computational Learning Theory, Pittsburgh, PA, USA, 27-29 July 1992; pp. 144-152.

36. Breiman, L. Random forests. Mach. Learn. 2001, 45, 5-32. [CrossRef]

37. Liaw, A.; Wiener, M. Classification and Regression by randomForest. R News 2002, 2, 18-22. 
38. Ilyas, M.; Fournier, R.; Othmani, A.; Nait-Ali, A. BiometricAccessFilter: A web control access system based on human auditory perception for children protection. Electronics 2020, 9, 361. [CrossRef]

39. Specht, D.F. Probabilistic neural networks. Neural Netw. 1990, 3, 109-118. [CrossRef]

40. Specht, D.F. Probabilistic neural networks and the polynomial Adaline as complementary techniques for classification. IEEE Trans. Neural Netw. 1990, 1, 111-121. [CrossRef]

41. Zhang, J.C.; Pu, R.L.; Yuan, L.; Huang, W.J.; Nie, C.W.; Yang, G.J. Integrating remotely sensed and meteorological observations to forecast wheat powdery mildew at a regional scale. IEEE J. STARS 2014, 7, 4328-4339. [CrossRef]

42. He, Y.; Li, X.L.; Shao, Y.N. Discrimination of varieties of apple using near infrared spectra based on principal component analysis and artificial neural network model. Spectrosc. Spect. Anal. 2006, 26, 850-853.

43. Tian, W.; Xu, L.; Chen, Z.; Shi, A. Multiple feature learning based on edge-preserving features for hyperspectral image classification. IEEE Access 2019, 7, 106861-106872. [CrossRef]

44. Bazi, Y.; Melgani, F. Toward an optimal SVM classification system for hyperspectral remote sensing images. IEEE Trans. Geosci. Remote Sens. 2006, 44, 3374-3385. [CrossRef]

45. Raza, A.; Benrabah, A.; Alquthami, T.; Akmal, M. A review of fault diagnosing methods in power transmission systems. Appl. Sci. 2020, 10, 1312. [CrossRef]

46. Wu, D.; He, Y.; Nie, P.C.; Cao, F.; Bao, Y.D. Hybrid variable selection in visible and near-infrared spectral analysis for non-invasive quality determination of grape juice. Anal. Chim. Acta 2010, 659, 229-237. [CrossRef]

47. Wong, W.T.; Shih, F.Y.; Liu, J. Shape-based image retrieval using support vector machines, Fourier descriptors and self-organizing maps. Inf. Sci. 2007, 177, 1878-1891. [CrossRef]

48. Zhang, M.; Qin, Z.; Liu, X.; Ustin, S.L. Detection of stress in tomatoes induced by late blight disease in California, USA, using hyperspectral remote sensing. Int. J. Appl. Earth Obs. 2003, 4, 295-310. [CrossRef]

(C) 2020 by the authors. Licensee MDPI, Basel, Switzerland. This article is an open access article distributed under the terms and conditions of the Creative Commons Attribution (CC BY) license (http://creativecommons.org/licenses/by/4.0/). 